ARTICLE

\title{
Catalytic activity of graphene-covered non-noble metals governed by proton penetration in electrochemical hydrogen evolution reaction
}

Kailong $\mathrm{Hu}^{1}$, Tatsuhiko Ohto ${ }^{2 凶}$, Yuki Nagata (iD) ${ }^{3}$, Mitsuru Wakisaka ${ }^{4,5}$, Yoshitaka Aoki (iD ${ }^{5,6}$, Jun-ichi Fujita ${ }^{1}$ \& Yoshikazu Ito (1) $1,5 \times$

Graphene-covering is a promising approach for achieving an acid-stable, non-noble-metalcatalysed hydrogen evolution reaction (HER). Optimization of the number of graphenecovering layers and the density of defects generated by chemical doping is crucial for achieving a balance between corrosion resistance and catalytic activity. Here, we investigate the influence of charge transfer and proton penetration through the graphene layers on the HER mechanisms of the non-noble metals $\mathrm{Ni}$ and $\mathrm{Cu}$ in an acidic electrolyte. We find that increasing the number of graphene-covering layers significantly alters the HER performances of $\mathrm{Ni}$ and $\mathrm{Cu}$. The proton penetration explored through electrochemical experiments and simulations reveals that the HER activity of the graphene-covered catalysts is governed by the degree of proton penetration, as determined by the number of graphene-covering layers.

\footnotetext{
${ }^{1}$ Institute of Applied Physics, Graduate School of Pure and Applied Sciences, University of Tsukuba, Tsukuba 305-8573, Japan. ${ }^{2}$ Graduate School of Engineering Science, Osaka University, 1-3 Machikaneyama, Toyonaka 560-8531, Japan. ${ }^{3}$ Max Planck Institute for Polymer Research, Ackermannweg 10, 55128 Mainz, Germany. ${ }^{4}$ Graduate School of Engineering, Toyama Prefectural University, 5180 Kurokawa, Imizu, Toyama 939-0398, Japan. ${ }^{5}$ PRESTO, Japan Science and Technology Agency, Saitama 332-0012, Japan. ${ }^{6}$ Faculty of Engineering, Hokkaido University, N13W8 Kita-ku, Sapporo 060-8628, Japan.

凶email: ohto@molectronics.jp; ito.yoshikazu.ga@u.tsukuba.jp
} 
H ydrogen $\left(\mathrm{H}_{2}\right)$ generation is essential in an efficient and eco-friendly society because $\mathrm{H}_{2}$ is a clean and versatile fuel with an energy density of $140 \mathrm{MJ} / \mathrm{kg}^{1}$, which is much higher than that of fossil fuels such as gasoline, natural gas, and coal $(\sim 50 \mathrm{MJ} / \mathrm{kg})$. Among various $\mathrm{H}_{2}$ generation technologies ${ }^{2-4}$, the electrolytic production of $\mathrm{H}_{2}$ from water is a promising method ${ }^{3,5}$ because it yields high-purity $\mathrm{H}_{2}(>95 \%)$ and emits no $\mathrm{CO}_{2}$. Polymer electrolyte membrane (PEM) water electrolysis is likely to be the next-generation technology for $\mathrm{H}_{2}$ production; it is more efficient than alkaline water electrolysis because of its $\mathrm{H}_{2}$ gas purity ( $>99.99$ vol.\% for PEM vs. 99.5 vol.\% for alkaline), faster dynamic response, larger current density $\left(\sim 5 \mathrm{~A} \mathrm{~cm}^{-2}\right.$ for PEM vs. $\sim 0.45 \mathrm{~A} \mathrm{~cm}^{-2}$ for alkaline), and higher discharge $\mathrm{H}_{2}$ pressure (30-76 bar for PEM vs. 30-40 bar for alkaline) $)^{3,6}$.

One of the challenges associated with the PEM electrolysers is the degradation of the cathode catalyst under acidic conditions. Platinum (Pt) has frequently been employed as the cathode catalyst because it exhibits a high electrochemical activity and is chemically stable even in strongly acidic environments. However, the scarcity and high cost of noble-metal catalysts limit the use of PEM electrolysers. Given this limitation, it is important to develop cost-efficient, acid-stable, noble-metal-free catalysts that possess high hydrogen evolution reaction (HER) activity comparable to $\mathrm{Pt}$ and are chemically tolerant to acidic conditions.

The main source of HER catalyst degradation in acidic media is corrosion. This corrosion can be suppressed through passivation or covering of the catalyst surface with chemically stable graphene layers $^{7-10}$. For example, graphene-encapsulated $\mathrm{Ni}, \mathrm{Fe}$, and $\mathrm{CoNi}$ alloy nanoparticles have been used in acidic electrolytes ${ }^{11-13}$. These encapsulated nanoparticles show sufficient tolerance against corrosion yet maintain their HER performance because the outermost graphene layers become HER-active owing to charge transfer from the underlying metal substrate ${ }^{13}$. However, regardless of the charge transfer effect, the performance of graphene-covered non-noble metal catalysts seems to be strongly dependent on the underlying metal substrate ${ }^{11-13}$. Here, a fundamental question is: how can the HER effectively occur with graphene layer covering, where the covering graphene blocks the catalytically active sites on the surfaces of the non-noble metal catalysts? Although the HER mechanism can be understood by the charge transfer ${ }^{13}$ and proton penetration through graphene ${ }^{14,15}$, this has not been evidenced by experiments. Therefore, it is crucial to determine how both the charge transfer and the proton penetration through graphene affect the HER mechanism of the graphene-covered non-noble metal catalysts.

In this study, we investigate the HER mechanisms of the proton penetration/charge transfer through the graphene-covering layers and their impact on the catalytic activity of graphene-covered nonnoble metal catalysts. We find that protons as well as molecular hydrogen $\left(\mathrm{H}_{2}\right)$ penetrate by bypassing the $\mathrm{N}$-dopant-induced defect sites, which makes the catalysts covered by $\mathrm{N}$-doped graphene electrochemically more active than those covered by nondoped graphene. Our results reveal that the charge transfer effect is not a dominant factor, while the ability of protons to penetrate graphene governs the HER activity of graphene-covered non-noble metal catalysts regardless of morphology and composition. This study provides new insights for the development of non-noble metal catalysts for electrochemical hydrogen production through graphene covering by balancing the contrasting properties of corrosion resistance and catalytic activity in acidic media.

\section{Results}

Impact of charge transfer and proton penetration on the catalytic activity of graphene-covered non-noble metal catalysts. To evaluate the effects of charge transfer and proton penetration on the graphene-covered non-noble metal catalysts, we prepared a graphene-covered $50-\mu \mathrm{m}$-thick $\mathrm{Cu}$ sheet as a less-active HER system and a $50-\mu \mathrm{m}$-thick $\mathrm{Ni}$ sheet as a HER-active system with different numbers of non-doped graphene layers (GLs) and Ndoped graphene layers (NGLs) (Fig. 1a and Supplementary Fig. 1). The graphene layers were prepared by a conventional chemical vapour deposition (CVD) method ${ }^{16}$ (see the Supplementary methods for details).

The crystallinities of graphene, various layer numbers, and degrees of structural defects were characterized by transmission electron microscopy (TEM), Raman spectroscopy, and X-ray photoelectron spectroscopy (XPS) ${ }^{17}$. The atomic structures of GLs and NGLs with various layer numbers were observed using highresolution transmission electron microscopy (HRTEM). The HRTEM image of the monolayer GL (1GL) showed a honeycomb structure comprising six carbon atoms with six-fold symmetry spots assigned to monolayer graphene (Fig. 1b). The bilayer and trilayer GL (2GLs and 3GLs) possessed randomly orientated sixfold symmetry spots; i.e. 12 spots for the 2 GLs and 18 spots for the 3GLs (Supplementary Fig. 2a, b) ${ }^{18,19}$. The HRTEM images of NGLs showed various types of topological defect (e.g. single and double vacancies) induced by N-doping (Inset of Fig. 1c, and Supplementary Fig. $2 c, d)^{20}$. Raman images of 1GL and 1NGL on a window-attached $\mathrm{Si}_{3} \mathrm{~N}_{4}$ chip (window size: $10 \times 10 \mu \mathrm{m}$ ) indicated high $2 D$-band to $G$-band $\left(I_{2 \mathrm{D}} / I_{\mathrm{G}}\right)$ intensity ratios of 3.8-4.9, suggesting that the monolayer graphene is highly crystalline $^{21,22}$ (Fig. 1d-g, Supplementary Figs. 3-9, and Supplementary Table 1). Raman images of the GLs showed low D-bandto- $G$-band $\left(I_{\mathrm{D}} / I_{\mathrm{G}}\right)$ intensity ratios of $0.05-0.08$. In contrast, the NGLs exhibited relatively high $I_{\mathrm{D}} / I_{\mathrm{G}}$ ratios (0.7-1.4), which illustrates that $\mathrm{N}$-doping induced many defects. We interrogated the chemical binding state of GLs and NGLs through XPS (Supplementary Fig. 10). The C 1s XPS spectra of the GLs and the NGLs confirmed the high quality of the graphene without evident oxidation. The N 1s XPS spectrum revealed that the $\mathrm{N}$-dopants exist in pyridinic (0.18 at.\%), graphitic (0.76 at.\%), and oxidic structures $\left(0.36\right.$ at.\%) in the NGLs ${ }^{23,24}$. We observed a very tiny amount of residual Cu metal ( $\leq 0.01$ at.\%) (Supplementary Fig. 11).

We subsequently transferred the graphene layers onto the $\mathrm{Cu}$ and Ni sheets with trilayer NGL and hexalayer NGL (abbreviated as $\mathrm{Cu} / 3 \mathrm{NGL}, \mathrm{Cu} / 6 \mathrm{NGL}, \mathrm{Ni} / 3 \mathrm{NGL}$, and $\mathrm{Ni} / 6 \mathrm{NGL}$, respectively). These graphene-covered $\mathrm{Ni}$ and $\mathrm{Cu}$ sheets were sandwiched between Nafion films. We measured the HER activities of the $\mathrm{Cu} /$ 3-6NGL and Ni/3-6NGL samples in $0.5 \mathrm{M} \mathrm{H}_{2} \mathrm{SO}_{4}$ using a threeelectrode system and compared them to those of the bare 3-6NGL samples (without metal substrates) as well as the bare $\mathrm{Cu}$ and $\mathrm{Ni}$ sheets (without graphene-covering). The HER polarization curves of the $\mathrm{Cu} / 3-6 \mathrm{NGL}$ samples showed that the overpotential $\left(\eta_{10}\right)$ at the current density $10 \mathrm{~mA} \mathrm{~cm}^{-2}$, normalized by the electrode surface area, increased with an increasing number of NGLs (Fig. 2a). The $\eta_{10}$ value of the $\mathrm{Cu} / 3$ NGL sample is $36.0 \%$ lower than that of the bare 3 NGL sample, which can be attributed to charge transfer from the underlying metal substrate to the outermost graphene layers ${ }^{13}$. As previously reported, the charge transfer effect becomes negligible beyond three graphenecovering layers $^{8,13}$. Indeed, the $\mathrm{Cu} / 6 \mathrm{NGL}$ exhibited a $6.5 \%$ lower $\eta_{10}$ value compared to the bare 6 NGL. This result suggests that the charge transfer effect determines the HER activity of $\mathrm{Cu} / \mathrm{NGL}$ samples ( $\mathrm{Cu}$ is HER-less-active).

We further measured the HER activities of the Ni/NGL samples under the same experimental conditions (Fig. 2b). If the entire HER occurs on the outermost graphene layers and the charge transfer solely determines the HER activity, the $\eta_{10}$ values of the $\mathrm{Cu} / \mathrm{NGL}$ and Ni/NGL samples should be identical regardless of the catalytic activity of the metal substrates. However, the observed $\eta_{10}$ values of the Ni/3NGL and $\mathrm{Ni}$ / 
a

Graphene-coated non-noble metals
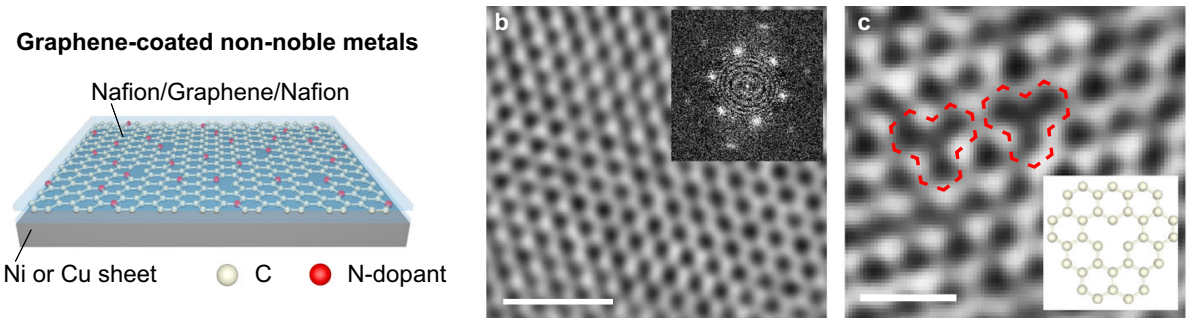

d
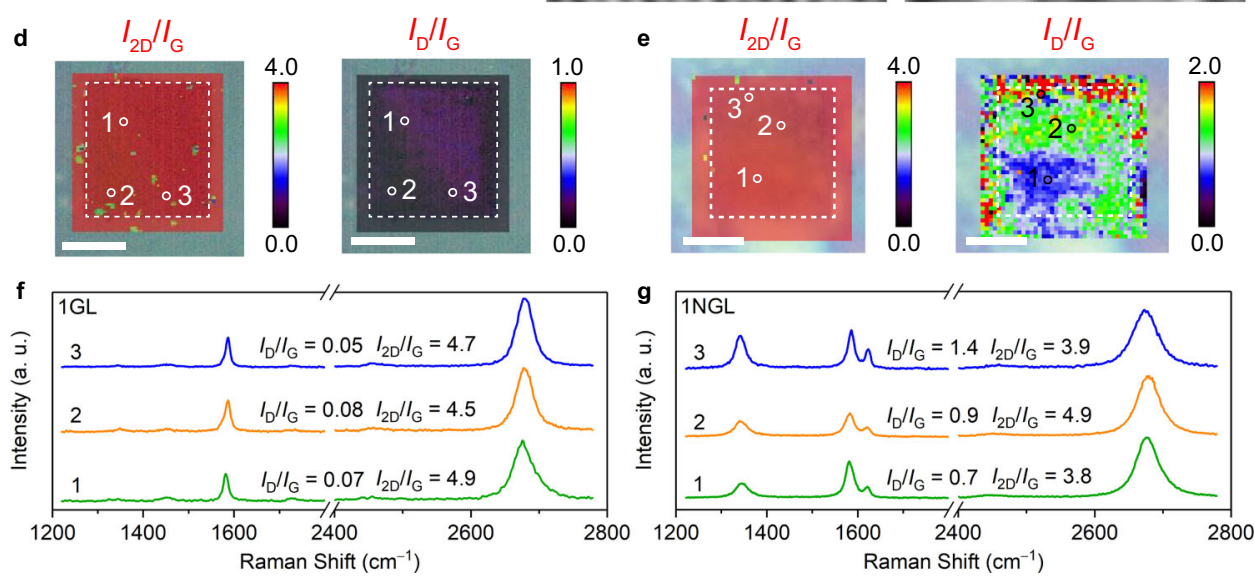

Fig. 1 Graphene-covered non-noble metal catalysts and graphene characterization. a Schematic diagram of a graphene-covered Cu or Ni sheet. HRTEM images of (b) $1 \mathrm{GL}$ and (c) 1NGL. Inset of (b): the corresponding fast Fourier transform (FFT) image. Inset of (c): the atomic model of the structural defect, highlighted by red marks. Raman maps of (d) $1 \mathrm{GL}$ and (e) $1 \mathrm{NGL}$ performed on a window-attached $\mathrm{Si}_{3} \mathrm{~N}_{4}$ chip. The dotted square indicates the window area. f, $\mathbf{g}$ Raman spectra were collected at the positions indicated on the corresponding maps. The $I_{2 \mathrm{D}} / I_{\mathrm{G}}$ values provide evidence that monolayer graphene dominates. Scale bars: (b) $1 \mathrm{~nm}$; (c) $0.5 \mathrm{~nm}$; (d, e) $5 \mu \mathrm{m}$.

6NGL samples were $53.3 \%$ and $29.7 \%$ lower than those of the bare 3 NGL and 6NGL samples. The reductions in the $\eta_{10}$ values due to the Ni substrate (53.3\% for the 3NGL and $29.7 \%$ for the 6 $\mathrm{NGL})$ are much larger than the reductions due to the $\mathrm{Cu}$ substrate (36.0\% for the 3NGL and $6.5 \%$ for the 6 NGL, Supplementary Table 2). This is also the case for the GL samples (Fig. 2c). The dramatic decrease in the $\eta_{10}$ values for the Ni/NGL samples cannot be solely explained by the effects of charge transfer. These differences in catalytic activity between the HER-less-active $\mathrm{Cu}$ and the HER-highly-active Ni suggest that the HER occurs not only on the graphene surface but also on the underlying metal surface.

To confirm that HER occurs on the covered metal surface, we applied an electrode potential to the Ni/3GL sample with a slow scan rate $(0.01 \mathrm{mV} / \mathrm{s})$ and observed the generation of $\mathrm{H}_{2}$ bubbles at the interface between the $\mathrm{Ni}$ sheet and the graphene layers (Fig. 2d). Raman spectra measured at the spot marked by the red star verified that the generation of the bubbles occurred at the interface and that there were no obvious cracks in the graphene layers (inset of Fig. 2d). Overall, our results strongly suggest that the protons penetrate through the graphene layers and reach the underlying metal substrate, triggering HER.

Electrochemical proton-penetration experiments. Above, we confirmed that the proton penetrates through the graphene layers. To study the mechanism of the proton penetration, GLs and NGLs were employed as separating membranes in an H-type cell (Fig. 3a). Monolayer GL and NGL were stacked layer-by-layer and covered with Nafion (proton conductivity: $\sim 90 \mathrm{mS} \mathrm{cm}^{-1}$ at $24^{\circ} \mathrm{C}^{25}$ ) on both sides. The Nafion/graphene layers/Nafion sandwich membrane was placed on the same $\mathrm{Si}_{3} \mathrm{~N}_{4}$ chip used for the Raman measurements to isolate the anode and cathode chambers of the H-type cell (Fig. 3a-c).
We subsequently examined the proton penetration pathway through graphene by measuring the open circuit potential $\left(E_{\mathrm{OC}}\right)$ between the two chambers that were physically separated by graphene in the H-type cell (Supplementary Fig. 12). The measured $E_{\mathrm{OC}}$ value remained constant at $71 \mathrm{mV}$ for $6 \mathrm{~h}$ at a $\mathrm{pH}$ of 1.74 in the anode chamber and a $\mathrm{pH}$ of 0.50 in the cathode chamber. The experimental value of $71 \mathrm{mV}$ is close to the theoretical $E_{\mathrm{OC}}$ value of $73 \mathrm{mV}$ calculated by the Nernst equation (see Supplementary methods), which confirms that a proton penetration pathway exists and that the electrolyte does not leak. We explored the degrees of the proton penetration through the GLs and NGLs from the current-voltage $(I-V)$ characteristics in a two-electrode system. The proton current through the graphene layers was proportional to the bias voltage in the +200 to -200 $\mathrm{mV}$ range (Fig. 3d and Supplementary Fig. 13). The measured proton currents varied linearly with the bias voltage and their slopes decreased gradually with an increasing number of graphene layers, suggestive of an increasing resistance to proton penetration. The proton conductivities through 1GL and 1NGL were 0.27 and $0.44 \mathrm{mS} \mathrm{cm}^{-2}$, respectively (Supplementary Figs. 14 and 15, and Supplementary Table 3), which were much smaller than the proton conductivity through a Nafion separator membrane $(43.0$ $\mathrm{mS} \mathrm{cm}^{-2}$ ). This manifests that the proton-penetration resistance arises predominantly from the graphene layers rather than the Nafion. The electrochemical resistance of proton penetration through the graphene layers was further investigated by electrochemical impedance spectroscopy. The results also confirmed the correlation of the proton-penetration resistance and the layer number of graphene (details in Supplementary discussion 1, Supplementary Fig. 16, and Supplementary Table 4).

Time dependence of the proton penetration at a constant electrode potential in a three-electrode system was investigated by chronoamperometry (CA). The proton currents, which are defined as the limited current of HER by proton diffusion, through the 

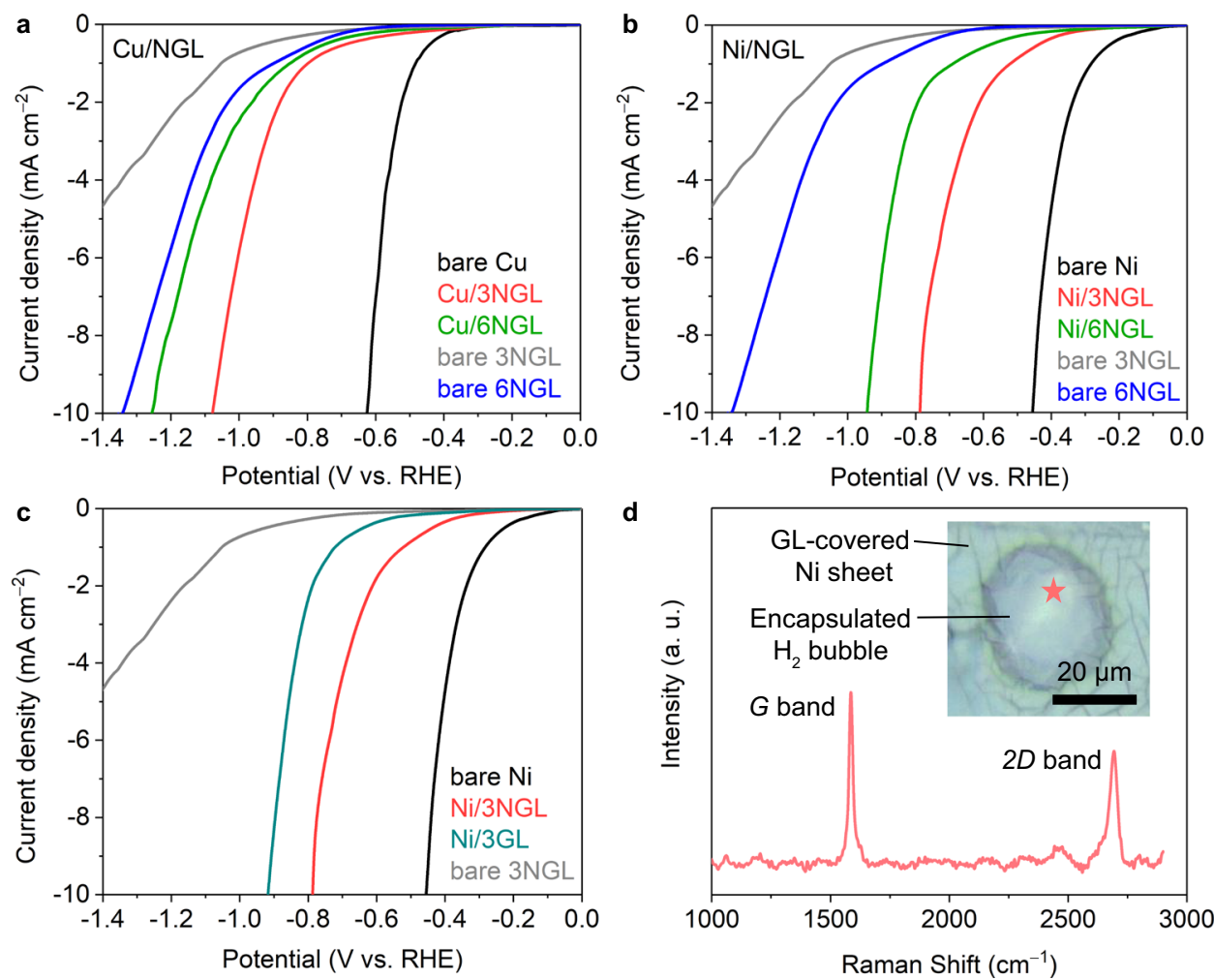

Fig. 2 HER activities of the graphene-covered $\mathbf{C u}$ and Ni sheets. HER polarization curves of the graphene-covered (a) Cu and (b, c) Ni sheets. $\mathbf{d}$ Raman spectra of the $3 \mathrm{GL}$ on a $\mathrm{H}_{2}$ bubble after the HER test. Inset of $(\mathbf{d})$ : the optical photo shows that a $\mathrm{H}_{2}$ bubble was generated between the Ni sheet and the graphene layers. The Raman measurement was obtained at the location indicated by the red star.

NGLs were observed to be 1.5 times greater than those through the GLs (Fig. 3e, f, Supplementary Figs. 17-19, and Supplementary Table 5). Eventually, we observed almost zero current for the 10layer graphene. This penetration behaviour was further investigated by isotope experiments (Supplementary Figs. 20 and 21, and Supplementary Tables 6 and 7). The proton current through 1GL was 1.4-1.7 times larger than the deuteron current. The difference in the current between proton and deuteron suggests the isotope effect is present (Supplementary Fig. 20).

We experimentally examined the energy barriers for proton penetration through graphene membranes by measuring the proton conductivity as a function of the temperature (Fig. $3 \mathrm{~g})^{14}$. An Arrhenius-type-fit of the data provides the energy barriers of $0.95 \pm 0.03$ and $0.87 \pm 0.03 \mathrm{eV}$ for $1 \mathrm{GL}$ and $1 \mathrm{NGL}$, respectively. In addition, the energy barriers for GLs are almost proportional to the number of GLs (2GL: $1.76 \pm 0.04 \mathrm{eV}$ ) while the energy barriers for NGLs are not simply proportional to the number of NGLs (3NGL: $1.17 \pm 0.02 \mathrm{eV}, 6$ NGL: $1.42 \pm 0.02 \mathrm{eV}$ ). These results indicate that the nitrogen dopant-induced defects on the graphene lattice accelerate the proton penetration ${ }^{26}$. Moreover, the $I-V$ curves of 6 NGL demonstrated a non-linear behaviour at a wide voltage range from -3.5 to $+3.0 \mathrm{~V}$ (Supplementary Fig. 22). The "apparent" activation energy at a high voltage range from -3.5 to $-3.0 \mathrm{~V}$ was estimated as $0.43 \pm 0.02 \mathrm{eV}$, which is much lower than the $1.42 \pm 0.02 \mathrm{eV}$ energy barrier estimated at a low voltage range from -0.2 to $0.0 \mathrm{~V}$. This manifests that the activation energy can be drastically lowered due to the applied voltage; the protons overcome the activation energy utilizing an applied electric field. In fact, the simulation shows that the high applied voltage primarily affects the electric potential near the graphene interface, with a thickness of sub-nanometre (Supplementary Fig. 23). Take these results together, we conclude that protons can penetrate through the graphene layers under an applied voltage, and this penetration is enhanced by $\mathrm{N}$-doping and by reducing the number of graphene layers.

Universal relationship between the proton current and HER activity. We correlated the proton penetration with the HER activities of the graphene-covered Ni sheet, Ni nanoparticle (NiNP), and NiMo alloy nanoparticle (NiMoNP) catalysts (Supplementary method, Supplementary discussion 2-6, Supplementary Figs. 24-35, and Supplementary Table 8). We plotted the average proton current density $\left(\mathrm{mA} \mathrm{cm}^{-2}\right)$ from $8 \mathrm{~h}$ of CA testing as a function of the overpotential $\left(\eta_{10-\text { tot }}\right)$ at the current density of $10 \mathrm{~mA} \mathrm{~cm}_{\text {tot }}-2$, normalized by the total surface area (i.e. the Brunauer-EmmettTeller (BET) surface area of the catalyst), and the current density at $-600 \mathrm{mV}$ vs. RHE for fair catalytic ability comparisons (Fig. 4 and Supplementary Fig. 32). Surprisingly, excellent correlations between the proton penetration through the graphene layers and the catalytic activity were observed. These results reveal that the catalytic activity of the graphene-covered non-noble metal catalysts is governed by proton penetration through the graphene layers, regardless of substrate morphology (sheet or nanoparticle) or composition (metal or alloy). Moreover, similar but shifted slopes were observed for the Ni sheet and NiNP samples, while the NiMoNP sample exhibited a shallower slope. This can be attributed to the number of active sites for the same substrate composition but with different morphology (Ni sheet vs. NiNP), and to the catalytic activity of the same substrate morphology but with different compositions (NiNP vs. NiMoNP). Thus, the HER overpotential of the graphene-covered non-noble metal catalysts reflects the HER activity of the underlying metal catalysts.

Unveiling proton-penetration pathways using density functional theory. Questions that arise here include: how do NGLs 

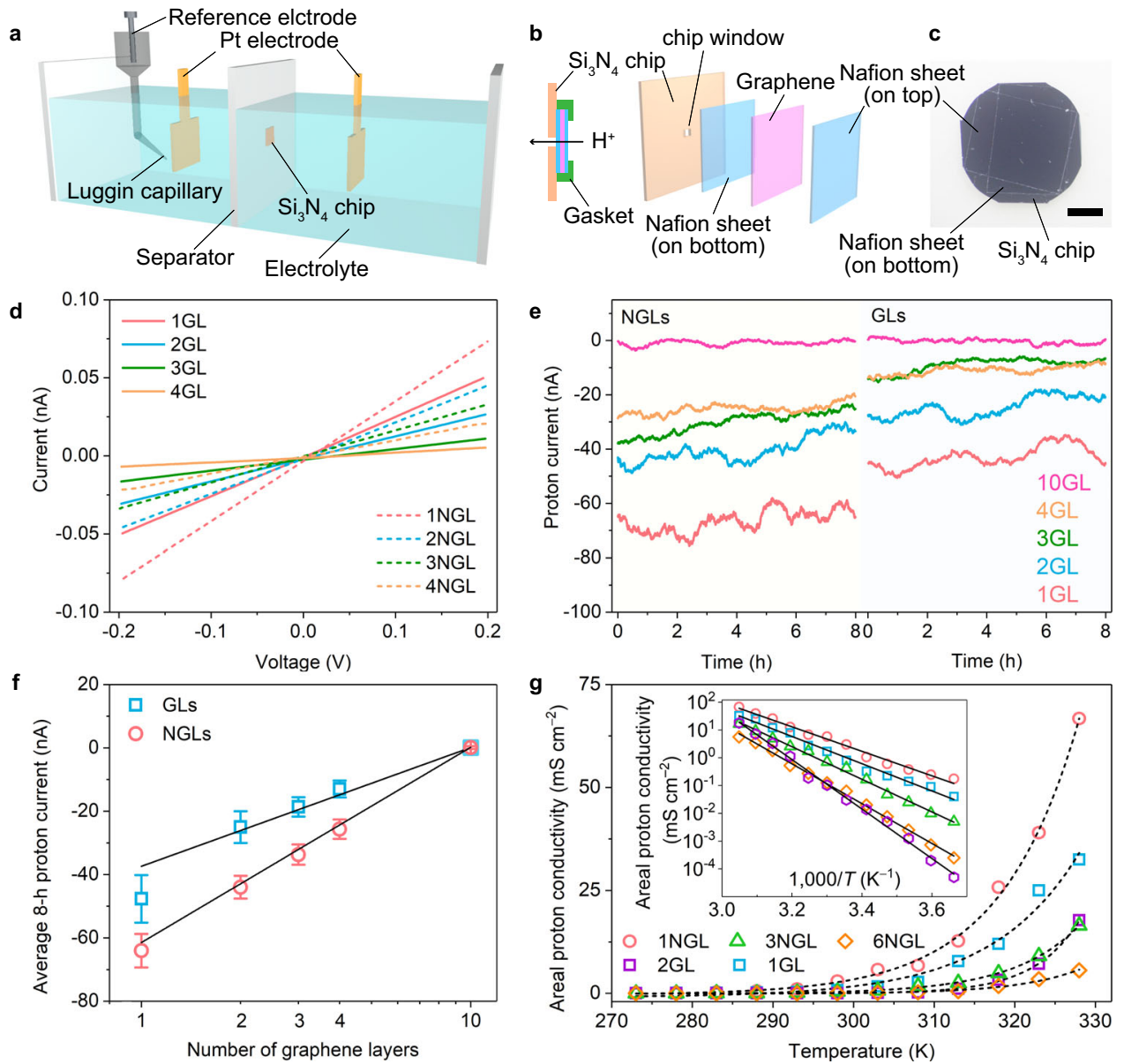

Fig. 3 H-type cell fabrication and electrochemical data for proton penetration through graphene layers. a Schematic of the H-type cell. b Placement of a stacked Nafion/graphene/Nafion sandwich membrane over the window area of a $\mathrm{Si}_{3} \mathrm{~N}_{4}$ chip. Nafion acts as a protective sheet for graphene layers with high proton conductivity, chemical/electrochemical stability, and extremely low electron conductivity 43 . All gaps and points of connection were sealed by an acid-stable, ion-impenetrable gasket to ensure that protons could only pass through the graphene on the window area. c Optical photographic image of the $\mathrm{Si}_{3} \mathrm{~N}_{4}$ chip with an attached Nafion/graphene/Nafion sandwich. $\mathbf{d} /-\mathrm{V}$ characteristics of the proton penetration through non- and $\mathrm{N}$-doped graphene membranes with one to four layers. The solid and dashed curves represent the proton currents observed through GLs and NGLs, respectively. e Proton currents collected by CA at a cathode potential of $-20 \mathrm{mV}$ vs. RHE through the GLs and the NGLs. $\mathbf{f}$ Average proton currents collected during $8 \mathrm{~h}$ of CA testing. Error bars show the fluctuations in the measured signals. $\mathbf{g}$ Temperature dependences of proton conductivity for 1GL, 2GL, 1NGL, 3NGL, and $6 \mathrm{NGL}$. Inset of $(\mathbf{g}): \log \sigma$ as a function of $T^{-1}$. The solid and dashed curves represent the fits to the experimental results.

enhance proton penetration compared to GLs, and how does a proton penetrate through multiple graphene layers? To address these questions, we computed the energy barriers for proton penetration through a defect-free 1GL lattice, a 1GL lattice with a 5-7 defect, and several types of NGL lattices using the nudged elastic band (NEB) method. Our calculations reveal energy barriers of 3.16 $\mathrm{eV}$ and $2.30 \mathrm{eV}$ for a $1 \mathrm{GL}$ lattice with no defects and a $1 \mathrm{GL}$ lattice with a 5-7 defect, respectively (Fig. 5a, Supplementary Figs. 36-38, and Supplementary Tables 9 and 10). The barrier is reduced because the proton can more easily penetrate through the seven-membered carbon rings in the defect-containing graphene than through the six-membered carbon rings in the defect-free graphene.

Pyridinic N-dopants also lowers the energy barrier for proton penetration. The pyridinic NGL lattice requires an energy barrier of $2.35 \mathrm{eV}$ for proton penetration through its six-membered carbon rings, which is $0.81 \mathrm{eV}$ lower than that of the defect-free GL lattice (Fig. 5a) and is ascribable to the electronegativity of the N-dopant (Supplementary Fig. 39). Proton penetration through NGL lattices containing a single vacancy (SV) and three $\mathrm{N}$-dopants $(\mathrm{SV}-3 \mathrm{~N})$ requires more energy than required for penetration through a defect-free GL lattice, because of stronger proton/ $\mathrm{N}$ binding (i.e. the protons tend to be trapped by $\mathrm{N}$ - dopants). However, the adsorption of $\mathrm{H}$ atoms at the $\mathrm{N}$-dopants reduces the barrier of proton penetration by contributing to proton repulsion. For example, the SV-3N lattice with one adsorbed $\mathrm{H}$ atom $(\mathrm{SV}-3 \mathrm{~N}-1 \mathrm{H})$ exhibits a smaller energy barrier $(2.65 \mathrm{eV})$ compared to that for the SV-3N lattice $(3.30 \mathrm{eV})$. The energy barrier of the SV-3N lattice decreases from $3.30 \mathrm{eV}$ to 1.93 $\mathrm{eV}$ in the presence of $\mathrm{H}_{2} \mathrm{O}$ molecules (Supplementary Fig. 38). Our results suggest that 5-7 topological defects and pyridinic Ndopants reduce the energy barrier for proton-penetration, compared with those of the defect-free graphene.

To determine how a proton penetrates through multiple graphene layers, we calculated the energy barriers for protonhopping between the bilayer SV-3N graphene lattices (Fig. 5b). As discussed above, a proton can be trapped by a pyridinic N-dopant. Such a trapped/adsorbed proton can hop to another pyridinic Ndopant in another graphene layer. The energy barrier for proton hopping depends highly on the relative positions of the pyridinic $\mathrm{N}$ dopants in the two layers $(1.56-4.10 \mathrm{eV})$. The energy barrier can be as low as $1.56 \mathrm{eV}$ when the two $\mathrm{N}$-dopants are close to each other; this barrier is smaller than that for pyridinic N-doped monolayer graphene (Fig. 5a). Our results show that a proton can penetrate through multiple graphene layers by hopping between $\mathrm{N}$-dopants. 


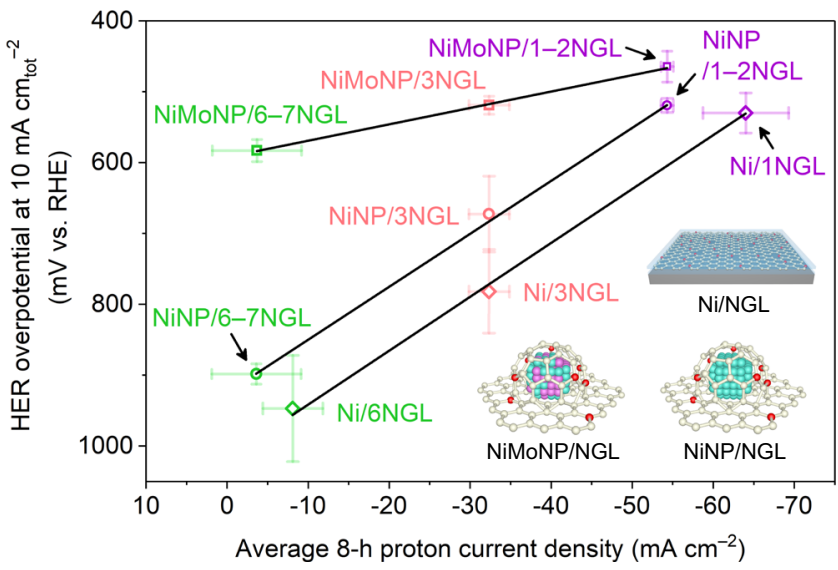

Fig. 4 Universal correlation between the HER activity and the proton penetration through the graphene layers. Graphene-covered $\mathrm{Ni}$ sheet, $\mathrm{NiNP}$, and NiMoNP with various numbers of graphene layers were employed. Colour code: carbon (white), nitrogen (red), nickel atom (cyan), nickel sheet (grey) and molybdenum (purple). Error bars show the fluctuations in the measured signals. The current density was normalized by the window area for the $X$-axis and by the total surface area of the catalysts for the $Y$-axis. Note: a $10 \mathrm{nA}$ proton current is equivalent to a 10 $\mathrm{mA} \mathrm{cm}-2$ proton current density.
The pathway for proton penetration through multiple graphene layers to the non-noble metal surface in the presence of $\mathrm{H}_{2} \mathrm{O}$ molecules is summarized in Fig. $5 \mathrm{c}$ and Supplementary Fig. 40. A proton is adsorbed on an N-dopant of the topmost layer (Step 1), and then the proton intercalates into the bilayer lattice (Step 2). As in Step 1, the proton is adsorbed onto the inner layer (Step 3). The proton finally reaches the NiMo surface (Steps 4 and 5; Supplementary Fig. 41) where the protons undergo $\mathrm{H}_{2}$ evolution. According to this sequence of events, adsorption on the NiMo substrate is energetically preferable to complete desorption following penetration (i.e. returning to the initial state requires an energy barrier of $2.00 \mathrm{eV}$ ).

Unveiling the ejection mechanism of the generated hydrogen molecules. We explored the ejection mechanism of generated $\mathrm{H}_{2}$ molecules through graphene lattices (Supplementary Figs. 42-46). The reported studies ${ }^{9-11}$ suggested that generated $\mathrm{H}_{2}$ bubbles cannot penetrate through a defect-free $\mathrm{GL}^{27}$. Indeed, we observed that the generated hydrogen bubbles were grown in the confined region sandwiched by the $\mathrm{Ni}$ sheet and the defect-less GLs at the low potential range from 0.0 to $-0.2 \mathrm{~V}$ (vs. RHE) (Supplementary Fig. 45b). Moreover, because of the growth of the $\mathrm{H}_{2}$ bubble, the crystallinity of the bubble encapsulating graphene was lowered (Supplementary Fig. 46b). Meanwhile, the defects and nanopores allowed the $\mathrm{H}_{2}$ molecule to move from the Ni surface to the a

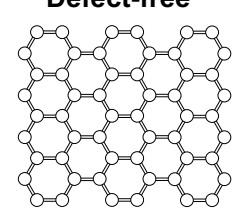

$3.16 \mathrm{eV}$

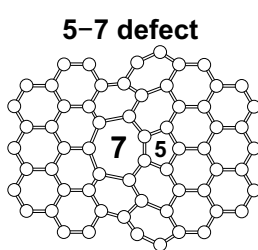

$-2.30 \mathrm{eV}$
Pyridinic $\mathbf{N}$

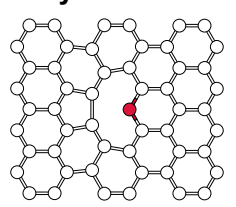

$2.35 \mathrm{eV}$
SV-3N

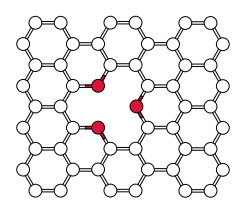

$-3.30 \mathrm{eV}$
SV-3N-1H

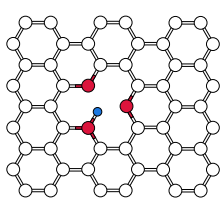

$-2.65 \mathrm{eV}$

b
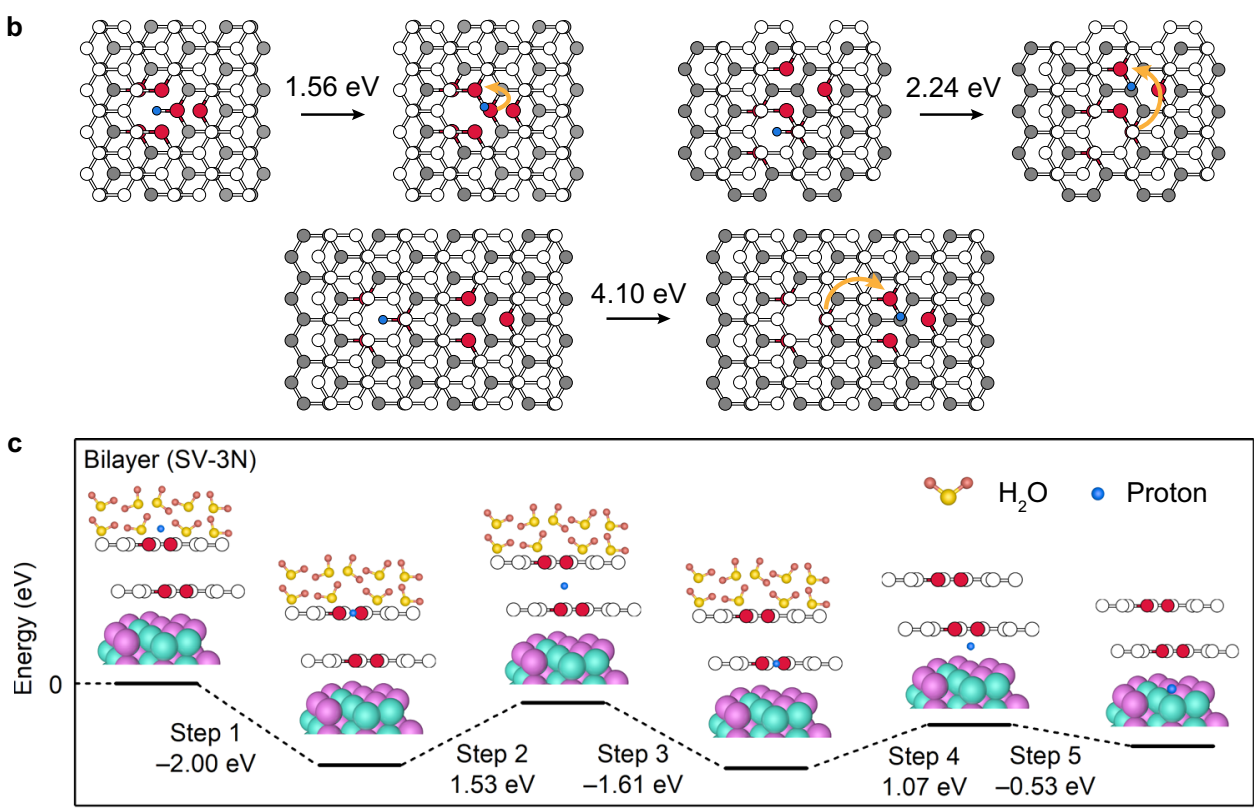

Reaction coordinate

Fig. 5 DFT calculations. a Atomic models of various types of graphene lattice. The numbers under the graphene lattices are the calculated energy barriers for proton penetration through the lattices. A positive or negative value indicates that the shape of the potential energy surface involves a peak or a trough (details in Supplementary Fig. 36). b Energy barriers for proton hopping between bilayer SV-3N graphene lattices (the first layer is white and the second layer is grey). Orange arrows indicate the hopping direction. c Energy diagram for proton penetration through a bilayer SV-3N graphene lattice. The proton was adsorbed on a NiMo nanoparticle after penetrating through the graphene-covering bilayer. Colour code: carbon (white), nitrogen (red), proton (blue), nickel (cyan), molybdenum (purple), oxygen (light yellow), and hydrogen (dark yellow). 
a Proton penetration type

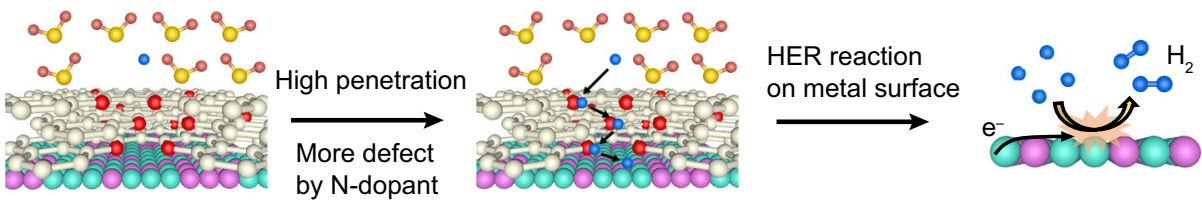

b Graphene surface reaction type

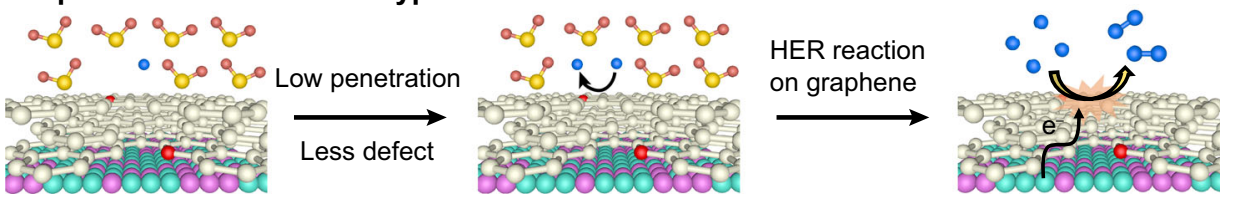

Fig. 6 Schematic HER mechanisms for graphene-covered non-noble metal catalysts. a Proton-penetration-type mechanism. Protons penetrate through the graphene covering layers, and $\mathrm{H}_{2}$ evolution occurs on the surface of the underlying non-noble metal substrate. b Graphene-surface-reaction-type mechanism. The $\mathrm{H}_{2}$ evolution occurs on the outermost graphene layer by utilizing charge transfer from the underlying non-noble metal substrate. Colour code: carbon (white), nitrogen (red), proton (blue), nickel (cyan), molybdenum (purple), oxygen (light yellow), and hydrogen (dark yellow).

outside of the graphene layers. These experimental results were further confirmed by the simulations. Our simulation data show that an $\mathrm{H}_{2}$ molecule must overcome an energy barrier of 4.6-5.4 $\mathrm{eV}$ to pass through defect-free graphene or graphene having a small topological defect (e.g. 5-7 or 5-8-5 defect), but it must only overcome an energy barrier of $<1.0 \mathrm{eV}$ when graphene has nanopores (diameter: 2.5-5.8 ) (Supplementary Table 11 and Supplementary discussion 7). Thus, the nanopores on graphene layers promote the ejection of generated $\mathrm{H}_{2}$ molecules. Note that the charge-neutral $\mathrm{H}_{2}$ penetrates more easily through defects/ nanopores than the excess number of protons and larger-sized hydrated cations ${ }^{28}$ at the cathodes. In other words, the hydrated ions are blocked from penetrating the graphene because additional energy is required for rearranging the solvation structure of ions and surrounding water molecules.

Nitrogen dopants and structural defects play an important role in $\mathrm{H}_{2}$ ejection. In the case of graphene/Ni sheet case, $\mathrm{H}_{2}$ bubbles were generated on the NGL-covered Ni sheet without the formation of cracks in N-doped graphene layers, which was opposite to the case of the GL-covered $\mathrm{Ni}$ sheet (Supplementary discussion 8 and Supplementary Figs. 44-46). These differences between the NGL and GL can be attributed to the efficient ejection of $\mathrm{H}_{2}$ through dopant-/defect-rich regions in the NGL. In the case of graphenecovered metal/alloy nanoparticle morphology, a high defect density, e.g. 7-, 8- or larger-membered carbon rings, on the highly curved graphene lattice is geometrically required for constructing the curved morphology to fit the graphene lattice with the nanoparticle surface ${ }^{29,30}$, which contributes to the $\mathrm{H}_{2}$ molecule ejection.

\section{Discussion}

We explored the impact of proton penetration on catalytic activity by combining HER catalytic-activity experiments of graphene-covered non-noble metal catalysts, proton-penetration characterizations, and DFT-calculated energy barriers for the penetration. The linear relationship between the required HER overpotential in the graphene-covered non-noble metal catalysts and the proton-penetration current through the graphene layers (Fig. 4) strongly suggests that catalytic activity is dominated by proton penetration through the graphene layers. Our DFT calculations revealed that NGLs exhibit enhanced proton penetration compared with GLs as a result of graphene-lattice deformation through $\mathrm{N}$-doping, as well as the electronegativity of the $\mathrm{N}$-atom dopant (Supplementary Fig. 39), which is in agreement with the experimental results (Fig. 1c). The overall process, which can be universally applied to various graphene-covered non-noble metal catalysts, is summarized in Fig. 6 a.
It has been reported that the catalytic HER reaction arises from the charge transfer effect occurring on the outermost graphene layer (Fig. 6b) ${ }^{31,32}$. This raises the question of whether the charge transfer effect or the proton-penetration process is dominant for the current HER reaction. To address this, we investigated the HER activities of the graphene-covered $\mathrm{Ni}$ and $\mathrm{Cu}$ sheets. If the catalytic reaction that occurs on the graphene surface can compete with the underlying metal's catalytic process achieved by proton penetration, the required overpotentials in the $\mathrm{Ni}$ and $\mathrm{Cu}$ systems are comparable. However, a much larger overpotential in the $\mathrm{Cu}$ system is required in comparison to the Ni system (Fig. 2a, b), which reveals that the process depicted in Fig. $6 \mathrm{~b}$ is a minor contributor due to the lower catalytic activity of graphene than that of the underlying metal, thus the process shown in Fig. 6a dominates for graphene-covered non-noble metal catalysts.

Graphene-covering technology is also important for ensuring the catalyst lifetimes of non-noble metal catalysts. The monolayer graphene-covered NiMoNP sample dissolves during $1000 \mathrm{CV}$ cycles (Supplementary Fig. 30), whereas the catalyst with multilayers of graphene endures 1000 CV cycles (Supplementary Fig. 31). These results confirm that protons reach the underlying metal surface and dissolve the non-noble metal catalysts. Moreover, corrosion resistance (in the form of high energy barriers) gradually increases and the degree of proton penetration decreases with increasing numbers of graphene layers; hence, HER activity is sacrificed for an improved catalyst lifetime (i.e. to protect non-noble metals in the acidic medium) resulting from the low quantity of penetrating protons.

In conclusion, our study revealed the fundamental HER mechanism of graphene-covered non-noble metal catalysts in acidic electrolytes; our results demonstrated that layer number, $\mathrm{N}$-doping, and structural defects in the covering graphene govern the degree of proton penetration and, consequently, the catalytic HER activity. These results indicate that engineering the thickness, doping level, and defect density of the graphene would allow for optimization of the HER activity of non-noble metal catalysts, while simultaneously suppressing their corrosion. Techniques that optimize these factors in the covering graphene would be crucial in improving the usability of non-noble metal catalysts not only for the HER in acidic media, but also for various circumstances that require the corrosion-proofing of non-noble metals.

\section{Methods}

Synthesis of monolayer graphene for graphene-covered samples and separating membranes. Monolayer GL and NGL were grown on a $\mathrm{Cu}$ foil $(99.8 \%, 25$ $\mu \mathrm{m}$ thickness, Alfa Aesar) via a conventional CVD method using $\mathrm{CH}_{4}(99.99 \%)$ and pyridine (Aldrich, $99.8 \%$, anhydrous) as carbon and nitrogen sources ${ }^{16,24}$. The 
$\mathrm{Cu}$ foil was loaded on a corundum boat and inserted into the centre of a quartz tube $(\varphi 30 \times \varphi 27 \times 1000 \mathrm{~mm})$ in a furnace. The tube was heated at $1000{ }^{\circ} \mathrm{C}$ for $1 \mathrm{~h}$ under an atmosphere of $\mathrm{H}_{2}(100 \mathrm{sccm}, 99.99 \%)$ and $\mathrm{Ar}(200 \mathrm{sccm}, 99.99 \%)$. Then, GL was grown with an additional flow of $\mathrm{CH}_{4}(20 \mathrm{sccm}, 99.995 \%)$ for $30 \mathrm{~min}$, while NGL was grown with an additional flow of $\mathrm{CH}_{4}(20 \mathrm{sccm})$ and pyridine $(0.5 \mathrm{~m}$ bar). The as-synthesized graphene on $\mathrm{Cu}$ foils after $\mathrm{Cu}$ dissolution in $0.25 \mathrm{M} \mathrm{Fe}$ $\left(\mathrm{NO}_{3}\right)_{3}$ solution was manually stacked to prepare graphene-covered $\mathrm{Cu}$ and $\mathrm{Ni}$ sheets and graphene separating membranes (details in Supplementary method and Supplementary Figs. 1,3).

Characterization of samples. Morphology and microstructure of as-synthesized graphene were characterized using a scanning electron microscope (SEM, HITACHI S-4300), transmission electron microscopes (TEM, JEOL JEM-2100 F and JEM-ARM200F), and equipped energy dispersive spectroscopy (EDS, SDD Type, Detection surface area $30 \mathrm{~mm}^{2}$, Solid angle $0.26 \mathrm{sr}$ ). Raman spectra were performed using a Renishaw InVia Reflex with an incident wavelength of $532 \mathrm{~nm}$. X-ray diffraction (XRD) was carried out using a D2 PHASER ( $\mathrm{Cu}$ Kal radiation; $\lambda=$ $1.5406 \AA$, BRUKER). Surface chemical states were studied by X-ray photoelectron spectroscopy (XPS, AXIS ultra DLD, Shimazu) with an Al Ka and X-ray monochromator.

Electrochemical measurements. An electrochemical workstation (Biologic, VSP300) was used for all electrochemical measurements. Catalytic activities of graphene-covered $\mathrm{Cu} / \mathrm{Ni}$ sheets and $\mathrm{Ni} / \mathrm{NiMo}$ nanoparticles samples were performed in a three-electrode system. As-prepared graphene-covered catalysts, a graphite rod, an $\mathrm{Ag} / \mathrm{AgCl}$ electrode, and $\mathrm{Ar}$-saturated $0.5 \mathrm{M} \mathrm{H}_{2} \mathrm{SO}_{4}$ served as the working electrode, counter electrode, reference electrode, and electrolyte, respectively. The potential was calculated with respect to RHE using the equation: $E$ $(\mathrm{RHE})=E(\mathrm{Ag} / \mathrm{AgCl})+0.0591 \times \mathrm{pH}+0.197$. The $\mathrm{pH}$ value of the electrolyte was recorded as $0.5-0.55$. The $\mathrm{Ag} / \mathrm{AgCl}$ reference electrode was calibrated using a $\mathrm{Pt}$ wire as the working electrode. The polarization curves were obtained using a sweep rate of $5.0 \mathrm{mV} \mathrm{s}^{-1}$. The electrode potential was automatically $i R$-compensated with the ohmic resistance.

Proton penetration measurements. The $I-V$ characteristics and electrochemical impedance spectra were performed in a two-electrode system. The schematic of the homemade H-type cell for electrochemical measurements of proton penetration was shown in Fig. 3a. A glass separator with a window-attached $\mathrm{Si}_{3} \mathrm{~N}_{4}$ chip (window size: $10 \times 10 \mu \mathrm{m}$ ) fixed in the centre was used to isolate the anode and cathode chambers filled with $0.5 \mathrm{M} \mathrm{H}_{2} \mathrm{SO}_{4}$. The distance between the electrode and separator was $1.0 \mathrm{~cm}$. The size of the Pt plate electrode was $1.0 \times 1.0 \mathrm{~cm}$. Electrochemical impedance spectra were recorded with the frequency ranging from $10^{6}$ to $100 \mathrm{~Hz}$. The CA measurements were carried out in a three-electrode system, in which an $\mathrm{Ag} / \mathrm{AgCl}$ electrode equipped with a salt bridge was used as the reference electrode. Temperature dependence of proton conductivity experiments for graphene layers were performed over a temperature range of 273-328 K to avoid water freezing and membrane damage due to thermal expansion.

DFT calculation. The DFT calculations were performed via the $\mathrm{CP} 2 \mathrm{~K}$ program ${ }^{33}$. The Becke-Lee-Yang-Parr (BLYP) ${ }^{34,35}$ exchange-correlation functional was used. We employed double-zeta valence plus polarization (DZVP) basis sets. The core electrons were described by the Goedecker-Teter-Hutter pseudopotential ${ }^{36}$. The real-space density cut-off was set to $320 \mathrm{Ry}$. The van der Waals correction was included via Grimme's D3 method ${ }^{37}$. The simulation cell lengths in the $x-, y-$, and $z$-directions were $25.56,24.595$, and $50 \AA^{38,39}$, respectively. The $\mathrm{C}-\mathrm{C}$ bond lengths of pristine graphene are $1.42 \AA$. The 5-7 defect was introduced according to the reported literature ${ }^{40}$. The nudged elastic band method ${ }^{41}$ was used with three intermediate states. The calculated barrier heights are independent of calculation methods and consistent with previous reports (Supplementary Tables 9-11). The atomic charges were calculated using the iterative Hirshfeld scheme (Hirshfeld-I ${ }^{42}$.

\section{Data availability}

The data that support the findings of this study are available from the corresponding authors upon reasonable request.

Received: 21 February 2020; Accepted: 4 December 2020;

Published online: 08 January 2021

\section{References}

1. Chi, J. \& Yu, H. Water electrolysis based on renewable energy for hydrogen production. Chin. J. Catal. 39, 390-394 (2018).

2. Haryanto, A., Fernando, S., Murali, N. \& Adhikari, S. Current status of hydrogen production techniques by steam reforming of ethanol: a review. Energy Fuels 19, 2098-2106 (2005)
3. Carmo, M., Fritz, D. L., Mergel, J. \& Stolten, D. A comprehensive review on PEM water electrolysis. Int. J. Hydrog. Energy 38, 4901-4934 (2013).

4. Ni, M., Leung, D. Y., Leung, M. K. \& Sumathy, K. An overview of hydrogen production from biomass. Fuel Process. Technol. 87, 461-472 (2006).

5. Ursua, A., Gandia, L. M. \& Sanchis, P. Hydrogen production from water electrolysis: current status and future trends. Proc. IEEE 100, 410-426 (2011)

6. Abbasi, R. et al. A roadmap to low-cost hydrogen with hydroxide exchange membrane electrolyzers. Adv. Mater. 31, 1805876 (2019).

7. Deng, J., Deng, D. \& Bao, X. Robust catalysis on 2D materials encapsulating metals: concept, application, and perspective. Adv. Mater. 29, 1606967 (2017).

8. Hu, K. L. et al. Graphene layer encapsulation of non-noble metal nanoparticles as acid-stable hydrogen evolution catalysts. ACS Energy Lett. 3, 1539-1544 (2018).

9. Ito, Y. et al. Cooperation between holey graphene and NiMo alloy for hydrogen evolution in an acidic electrolyte. ACS Catal. 8, 3579-3586 (2018).

10. Haslam, G. E., Chin, X.-Y. \& Burstein, G. T. Passivity and electrocatalysis of nanostructured nickel encapsulated in carbon. Phys. Chem. Chem. Phys. 13, 12968-12974 (2011)

11. Tavakkoli, M. et al. Single-shell carbon-encapsulated iron nanoparticles: synthesis and high electrocatalytic activity for hydrogen evolution reaction. Angew. Chem. Int. Ed. 127, 4618-4621 (2015).

12. Wang, S. et al. Molybdenum-carbide-modified nitrogen-doped carbon vesicle encapsulating nickel nanoparticles: a highly efficient, low-cost catalyst for hydrogen evolution reaction. J. Am. Chem. Soc. 137, 15753-15759 (2015)

13. Deng, J., Ren, P., Deng, D. \& Bao, X. Enhanced electron penetration through an ultrathin graphene layer for highly efficient catalysis of the hydrogen evolution reaction. Angew. Chem. Int. Ed. 54, 2100-2104 (2015).

14. $\mathrm{Hu}, \mathrm{S}$. et al. Proton transport through one-atom-thick crystals. Nature 516, 227-230 (2014).

15. Mogg, L. et al. Perfect proton selectivity in ion transport through twodimensional crystals. Nat. Commun. 10, 1-5 (2019).

16. Li, X. et al. Large-area synthesis of high-quality and uniform graphene films on copper foils. Science 324, 1312-1314 (2009).

17. Jin, Z., Yao, J., Kittrell, C. \& Tour, J. M. Large-scale growth and characterizations of nitrogen-doped monolayer graphene sheets. ACS Nano 5 , 4112-4117 (2011).

18. Zan, R., Bangert, U., Ramasse, Q. \& Novoselov, K. Imaging of Bernal stacked and misoriented graphene and boron nitride: experiment and simulation. $J$. Microsc. 244, 152-158 (2011).

19. Zhao, H. et al. Growth and Raman spectra of single-crystal trilayer graphene with different stacking orientations. ACS Nano 8, 10766-10773 (2014).

20. Lin, Y. C. et al. Structural and chemical dynamics of pyridinic-nitrogen defects in graphene. Nano Lett. 15, 7408-7413 (2015).

21. Sun, Z. et al. Growth of graphene from solid carbon sources. Nature 468 , 549-552 (2010).

22. Hao, Y. et al. Probing layer number and stacking order of few-layer graphene by Raman spectroscopy. Small 6, 195-200 (2010).

23. Kondo, T. et al. Atomic-scale characterization of nitrogen-doped graphite effects of dopant nitrogen on the local electronic structure of the surrounding carbon atoms. Phys. Rev. B 86, 035436 (2012).

24. Ito, Y. et al. Chemical vapor deposition of $\mathrm{N}$-doped graphene and carbon films: the role of precursors and gas phase. ACS Nano 8, 3337-3346 (2014).

25. Liu, L., Chen, W. \& Li, Y. An overview of the proton conductivity of nafion membranes through a statistical analysis. J. Membr. Sci. 504, 1-9 (2016).

26. Griffin, E. et al. Proton and Li-ion permeation through graphene with eightatom-ring defects. Acs Nano 14, 7280-7286 (2020).

27. Sun, P. Z. et al. Limits on gas impermeability of graphene. Nature $\mathbf{5 7 9}$, 229-232 (2020).

28. Tielrooij, K., Garcia-Araez, N., Bonn, M. \& Bakker, H. Cooperativity in ion hydration. Science 328, 1006-1009 (2010).

29. Ito, Y. et al. Correlation between chemical dopants and topological defects in catalytically active nanoporous graphene. Adv. Mater. 28, 10644-10651 (2016).

30. Chen, L. et al. Heavily doped and highly conductive hierarchical nanoporous graphene for electrochemical hydrogen production. Angew. Chem. Int. Ed. 130, 13486-13491 (2018)

31. Jia, Y. et al. Defect graphene as a trifunctional catalyst for electrochemical reactions. Adv. Mater. 28, 9532-9538 (2016).

32. Jiao, Y., Zheng, Y., Davey, K. \& Qiao, S. Z. Activity origin and catalyst design principles for electrocatalytic hydrogen evolution on heteroatom-doped graphene. Nat. Energy 1, 1-9 (2016).

33. Kühne, T. D. et al. СР2K: An electronic structure and molecular dynamics software package - Quickstep: Efficient and accurate electronic structure calculations. J. Chem. Phys. 152, 194103 (2020).

34. Becke, A. D. Density-functional exchange-energy approximation with correct asymptotic behavior. Phys. Rev. A 38, 3098 (1988). 
35. Lee, C., Yang, W. \& Parr, R. G. Development of the Colle-Salvetti correlationenergy formula into a functional of the electron density. Phys. Rev. B 37, 785 (1988).

36. Goedecker, S., Teter, M. \& Hutter, J. Separable dual-space Gaussian pseudopotentials. Phys. Rev. B 54, 1703 (1996).

37. Grimme, S., Antony, J., Ehrlich, S. \& Krieg, H. A consistent and accurate ab initio parametrization of density functional dispersion correction (DFT-D) for the 94 elements H-Pu. J. Chem. Phys. 132, 154104 (2010).

38. Ohto, T., Tada, H. \& Nagata, Y. Structure and dynamics of water at water-graphene and water-hexagonal boron-nitride sheet interfaces revealed by ab initio sum-frequency generation spectroscopy. Phys. Chem. Chem. Phys. 20, 12979-12985 (2018).

39. Tocci, G., Joly, L. \& Michaelides, A. Friction of water on graphene and hexagonal boron nitride from ab initio methods: very different slippage despite very similar interface structures. Nano Lett. 14, 6872-6877 (2014).

40. Jeong, B. W., Ihm, J. \& Lee, G. D. Stability of dislocation defect with two pentagon-heptagon pairs in graphene. Phys. Rev. B 78, 165403 (2008).

41. Henkelman, G., Uberuaga, B. P. \& Jónsson, H. A climbing image nudged elastic band method for finding saddle points and minimum energy paths. $J$. Chem. Phys. 113, 9901-9904 (2000).

42. Bultinck, P., Alsenoy, V. C., Ayers, P. \& Carbó-Dorca, R. Critical analysis and extension of the Hirshfeld atoms in molecules. J. Chem. Phys. 126, 144111 (2007).

43. Mauritz, K. A. \& Moore, R. B. State of understanding of Nafion. Chem. Rev. 104, 4535-4586 (2004)

\section{Acknowledgements}

The authors thank Ms. Kazuyo Omura at the Institute for Material Research in Tohoku University for the XPS measurements. This work was sponsored by JST-PRESTO 'Creation of Innovative Core Technology for Manufacture and Use of Energy Carriers from Renewable Energy' (JPMJPR1541, JPMJPR1444, JPMJPR1341); the Toyota Mobility Foundation (TMF); JSPS Grant-in-Aid for Scientific Research on Innovative Areas 'Discrete Geometric Analysis for Materials Design': Grant Numbers JP20H04628, JP20H04639 and JP17H06460 (steering group); JSPS KAKENHI Grant Numbers JP18K14174, JP19K15505 and JP20K22546; NIMS microstructural characterisation platform as a program of 'Nanotechnology Platform Project', MEXT, Japan (Grant Number: JPMXP09A19NM0033); the TEPCO Memorial Foundation; Foundation for Promotion of Material Science and Technology of Japan (MST Foundation); Grand for Basic Science Research Projects from The Sumitomo Foundation; Iketani Science and Technology Foundation; JACI Prize for Encouraging Young Researcher; University of Tsukuba Basic Research Support Program Type S; and a cooperative program (Proposal No. 20G0002) of the CRDAM-IMR, Tohoku University. Y.N. expresses thanks for the financial support from the MaxWater initiative of the Max Planck Society. K.L.H. acknowledges the Japanese government MEXT scholarship. We used the supercomputer of ACCMS, Kyoto University, for DFT calculations.

\section{Author contributions}

Y.I. conceived the research idea. K.L.H. carried out the experiments. T.O. performed the DFT calculations. K.L.H., T.O., Y.N. and Y.I. wrote the manuscript. M.W., Y.A. and J.F. contributed to the fruitful discussions and revisions.

\section{Competing interests}

The authors declare no competing interests.

\section{Additional information}

Supplementary information is available for this paper at https://doi.org/10.1038/s41467 020-20503-7.

Correspondence and requests for materials should be addressed to T.O. or Y.I.

Peer review information Nature Communications thanks the anonymous reviewers for their contribution to the peer review of this work. Peer reviewer reports are available.

Reprints and permission information is available at http://www.nature.com/reprints

Publisher's note Springer Nature remains neutral with regard to jurisdictional claims in published maps and institutional affiliations.

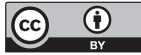

Open Access This article is licensed under a Creative Commons Attribution 4.0 International License, which permits use, sharing, adaptation, distribution and reproduction in any medium or format, as long as you give appropriate credit to the original author(s) and the source, provide a link to the Creative Commons license, and indicate if changes were made. The images or other third party material in this article are included in the article's Creative Commons license, unless indicated otherwise in a credit line to the material. If material is not included in the article's Creative Commons license and your intended use is not permitted by statutory regulation or exceeds the permitted use, you will need to obtain permission directly from the copyright holder. To view a copy of this license, visit http://creativecommons.org/ licenses/by/4.0/.

(C) The Author(s) 2021 